\title{
UN SALTERIO MANOSCRITTO DI PROVENIENZA OLIVETANA IN POLONIA
}

\section{Sommario}

L'articolo presenta i risultati della ricerca sul Salterio manoscritto, di provenienza olivetana, conservato nel Museo dell'Arcidiocesi di Łódź. L'autore fornisce una descrizione sintetica del codice, verifica il momento della sua creazione, specifica l'area geografica in cui è stato redatto e indica il luogo ipotetico del suo utilizzo. Alla luce di un'analisi comparativa del Salterio con altri manoscritti olivetani, dimostra che è stato scritto da un olivetano che era anche l'autore delle decorazioni del codice. La parte finale dell'articolo propone una ipotesi relativa all'arrivo del Salterio dall'Italia in Polonia.

Parole chiave: salterio; olivetani; Monte Oliveto; Łódź; officium divinum

$* * * * *$

Il Salterio, nel senso di libro liturgico di Officium Divinum, è una raccolta di 150 salmi disposti secondo un ciclo giornaliero e settimanale. Il libro così organizzato veniva chiamato salterio feriale o corale, liturgico. Nel tardo medioevo era un completamento dell'antifonario, contenendo le parti immutabili dell'Officium, cioè i testi e le melodie di salmi, invitatori, antifone, cantici, letture e responsori ${ }^{1}$. Questo tipo di libro è rappresentato dal manoscritto salterio di provenienza olivetana presente nella collezione del Museo dell'Arcidiocesi di Łódź (numero di ri-

* Ks. Piotr Wiśniewski - dr. hab., prof. KUL, Instytut Nauk o Sztuce, Katolicki Uniwersytet Lubelski Jana Pawła II

e-mail: piotr.wisniewski@kul.pl

https://orcid.org/0000-0001-8225-7552

${ }^{1}$ J. Bisztyga, Psalterium Andrzeja Piotrkowczyka z 1599 roku jako pierwszy dokument potrydenckiego choralu gregoriańskiego w Polsce, Lublin 2009, pp. 148, 222 (tesi di dottorato); Nella pratica liturgica e musicale integrava i libri dell'Officium usati nel Temporale, sull'esempio dell'Ordo, costituendo una sorta di supplemento ai libri di messa, e Commune Sanctorum per Sanctorale: A. Hughes, Medieval Manuscripts for Mass and Office. A guide to their organization and terminology, Toronto 1982, p. 226. 
ferimento MAŁ 357/ST/11), probabilmente l'unico Codice olivetano in Polonia ${ }^{2}$. Questo fatto da solo giustifica la necessità di intraprendere ricerche sul volume, di determinare il tempo e il luogo della sua produzione e di cercare di ricostruire le circostanze per cui il libro è giunto in Polonia. Purtroppo il Museo non dispone di documentazione su questo manoscritto. La breve descrizione bibliotecaria allegata al volume riporta solo che si tratta di un salterio cinquecentesco di provenienza monastica su pergamena nell'ordinamento dell'officium del breviario. Queste informazioni, tuttavia, richiedono una verifica in quanto il codice non è ancora stato introdotto nella circolazione scientifica. Resta da chiarire anche l'istituzione della collezione di manoscritti olivetani di cui fa parte il codice di Łódź. Il ritrovamento di questo libro, quindi, spinge a intraprendere una ricerca delle fonti volta a spiegare - per quanto possibile - tutte le problematiche sopra menzionate. Lo scopo di questo studio, che rappresenta il passo successivo nello studio del Salterio $^{3}$, è risolvere la datazione del codice, indicare del luogo in cui è stato creato e spiegare come esso sia divenuto proprietà del Museo di Łódź.

\section{Descrizione sintetica della fonte}

A seguito dei lavori di restauro effettuati nel 2020 a Cracovia, il Salterio ha ricevuto una nuova sovraccoperta, conservando gli elementi metallici originali che fanno parte della copertina. La rilegatura da $550 \times 405 \mathrm{~mm}$ (restaurata) è composta da due tavole rivestite in pelle marrone scuro, fissate con due cinture di pelle finite con fibbie $(\text { fibula })^{4}$.

Nome del codice: Il codice non ha un frontespizio separato. L'incipit del libro: „Psalterium secundum consuetudinem monacorum ordinis monasticis oliveti" indica che si tratta di un salterio olivetano. Ciò è confermato dal contenuto liturgico e musicale del libro. Nessun colofon.

Struttura del codice: Il codice ha 128 fogli di pergamena con dimensioni di $548 \times 385$ $\mathrm{mm}$, scritte su entrambi i lati, in 16 fascicoli di quaternioni regolari. I fascicoli sono contrassegnati con reclamanti, l'ultimo dei quali indica che il penultimo fascicolo manca dal codice. La pergamena utilizzata per il volume è una tipica charta

\footnotetext{
${ }^{2}$ Secondo l'opinione di Mauro Tagliabue, G.M. Canova, Corrispondenza e-mail (29.01.2021).

${ }^{3}$ Finora l'autore ha effettuato solo una revisione iniziale del codice e ha impostato le basi per ulteriori ricerche: P. Wiśniewski, Nieznany rękopis liturgiczno-muzyczny z Muzeum Archidiecezji Łódzkiej, „Muzyka” (2021), in stampa; L'articolo presenta i risultati delle ricerche successive, derivanti da ricerche nazionali ed estere.

${ }^{4}$ Nella sua forma originale, il codice era incorniciato in due tavole della stessa dimensione, $575 \times 400 \mathrm{~mm}$, ricoperte di velluto. I risguardi delle tavole erano coperti con fogli di carta. I bordi del libro erano fissati con raccordi angolari in metallo, che costituivano l'unico elemento decorativo esterno. Il libro era fissato con due fibbie, di uno di essi è stato conservato solo un frammento. Il dorso del codice era esposto e i fascicoli legati con sei spesse corde di canapa.
} 
Ordinamento della carta:

Notazione:

Scrittura latina: Decorazione:

Contenuto: theutonica $^{5}$ (pelle spessa, levigata su entrambi i lati). Il formato del codice deve essere specificato come in maior forma - forma regalis ${ }^{6}$. Il libro non ha foliazione. Il codice ha l'ordinamento della carta a due colonne (23 righe di testo), separate da un margine (intercolonna). Fanno eccezione sono 11 carte, sulle quali i contenuti (invitatori e suffragia) sono scritti in una sola colonna. Nota quadrata; linee a quattro righe redatte con inchiostro rosso, due tipi di chiavi: $c$ e $F$. Rotunda italiana (Gotico italiano). Il codice non ha miniature dipinte. È decorato con iniziali scritte a penna di varie dimensioni: semplici e filigranate, e alcune iniziali miniate con germogli di vite bianchi caratteristici dei manoscritti italiani (del territorio centrale e settentrionale) redatti intorno alla metà del XV secolo ${ }^{7}$. Tutte le iniziali sono scritte con inchiostro di due colori: blu e rosso. La prima pagina del codice è completamente circondata da un bordo floreale.

I. Horae minores ad Primam (dal lunedì al sabato);

II. Matutinum di domenica (tre notturni) e Laudes;

III. Matutinum e Laudes per i giorni feriali (dal lunedì al sabato);

IV. Horae minores (Prima, Tertia, Sexta, Nona);

V. Vesperae (solo un frammento dei primi Vespri della domenica);

VI. Completorium (preceduto da Collatio $^{8}$, incompleto); VII. Suffragia.

Nessuna raccolta degli inni separata ${ }^{9}$.

${ }^{5}$ W. Semkowicz, Paleografia tacińska, Kraków 2002, p. 53.

${ }^{6}$ A. Birkenmajer et al. (ed.), Encyklopedia wiedzy o ksiażce, Wrocław 1971, col. 723.

${ }^{7}$ R. Sosnowski, P. Tylus, Co mówia stare rękopisy, Kraków 2010, p. 89.

${ }^{8}$ Una breve lettura spirituale che si è svolta in refettorio, sala capitolare o chiostro, J. Kopeć, Collatio, in: Encyklopedia katolicka, vol. 3, ed. R. Łukaszyk, L. Bieńkowski, F. Gryglewicz, Lublin 1979, col. 540. Era un rito domenicano risalente al tempo di San Domenico (Liber Consuetudinum, Archiv für Litteratur und Kirchengeschichte des Mittelalters, 1885, vol. 1, pp. 199-200). Secondo l'usanza monastica, nei giorni quaresimali la compieta iniziava già in refettorio. Mentre i monaci si riunivano, il lettore chiedeva una benedizione e poi benediceva il vino diluito con il pasto serale. Poi iniziava la lettura spirituale, la collatio. Al segnale del priore, la lettura si concludeva con le parole: Tu autem Domine miserere nostri. Amen. Dopo i monaci passavano in processione silenziosa alla chiesa per il Confiteor e al resto dell'ufficio: vedi W. Bonniwell, Historia liturgii dominikańskiej 1215-1945, trad. D. Krupińska, New York 1945, www.rytdominikanski.pl/historia-liturgii-dominikanskiej/historia-liturgiidominikanskiej-1215-1945-o-william-bonniwell-op (accesso: 20.11.2020).

${ }^{9} \mathrm{Nel}$ Medioevo c'era una sorta di libertà nella scelta del posto degli inni nell'Officium Divinum. Soltanto con il Breviarium romanum di Pio V del 1658 gli inni vennero spostati nelle Lodi mattutine e nei Vespri dopo il capitulum e nelle ore rimanenti prima dei salmi. Le prime raccolte di inni sono 
Condizioni di conservazione: Il codice è in buone condizioni. L'inchiostro mantiene una buona saturazione nella scrittura liturgica, musicale e nella decorazione del libro. Le tracce dell'uso del libro sono visibili sotto forma di vari sporchi della diversa intensità, piccoli strappi o rughe di pergamena. L'ultima carta del quattordicesimo fascicolo è tagliata a metà.

\section{Datazione e provenienza}

Datare e individuare il luogo di produzione di un manoscritto non è un compito facile, ma il tipo di scrittura latina utilizzata costituisce un elemento che può indicare con precisione la sua origine. Lo stile di scrittura del Salterio di Łódź indica chiaramente il cosiddetto periodo gotico, dal XIII al XV secolo ${ }^{10}$. È in questo periodo infatti, quando la scrittura gotica si sviluppò in varie forme, che dovrebbe datarsi la creazione del manoscritto olivetano.

Nelle diverse aree geografiche la scrittura ha assunto un carattere diverso e individuale. Nelle varianti settentrionali era più slanciata, più o meno monumentale, ricca di punte aguzze, mentre nelle varianti meridionali prendeva una forma più tonda. Ecco perché la scrittura gotica in Italia è spesso chiamata rotunda (littera Bonaniensis, littera Venetiana $)^{11}$, dove tende a essere più larga, avendo la forma più morbida della rottura delle maniglie e affilatura delle curve. Come sottolineato da W. Semkowicz, le particolarità della rotunda includono la lettera -a- con un ventre basso e un arco profondamente disegnato; la lettera - $\mathrm{g}$ - a volte con la parte superiore a forma di trapezio e le lettere $\mathrm{f}, \mathrm{m}, \mathrm{n}, \mathrm{r}$, che hanno le gambe tagliate orizzontalmente sulla linea di fondo ${ }^{12}$. Già la destinazione d'uso del libro in esame ("monacorum ordinis monasticis oliveti") richiama l'attenzione sulla scrittura italiana. A un esame più attento, tenendo conto delle caratteristiche delle lettere indicate da Semkowicz, dovrebbe essere descritta come la rotunda italiana ed è esattamente questa la forma che rivelano le lettere del codice di Łódź. Le lettere piatte e larghe con archi delicatamente affilati e manici delicatamente spezzati consentono di classificare la scrittura del Salterio nel cosiddetto gotico italiano.

Determinare lo scriptorium in cui è stato redatto il Salterio non è una questione molto difficile. In primo luogo, occorre chiedersi se esistesse un modello olivetano per la creazione di libri liturgici e musicali, o Monte Oliveto era un modello in questo senso anche per i suoi altri monasteri? È noto che dalla metà del XIV secolo gli olivetani, come giovane comunità religiosa, preparavano essi stessi i libri liturgici necessari al servizio di Dio, come testimoniano i documenti. Tuttavia, non erano sempre anche i miniaturisti degli stessi libri, come evidenzia nella sua

note anche precedentemente alla formazione del breviario nel XIII secolo. Quando, nel XIII secolo, si iniziarono a realizzare i libri compatti per il canto dell'ufficio, queste raccolte entrarono a far parte del breviario. Nonostante ciò, furono preparate anche le raccolte di inni separati fino al XVIII secolo: vedi I. Pawlak, Hymnarz, in: Encyklopedia katolicka, vol. 6, ed. J. Walkusz, Lublin 1993, col. 1364-1365.

\footnotetext{
${ }^{10}$ Semkowicz, Paleografia tacińska, p. 313 nn.

${ }^{11}$ Ibidem.

${ }^{12}$ Ibidem, p. 320.
} 
ricerca G.M. Canova. Per acquisire le capacità di scrivere e decorare i manoscritti, gli olivetani stabilivano contatti con l'ambiente artistico e a volte impiegavano anche artisti laici qualificati per ornamenti più sofisticati ${ }^{13}$. Considerando $i$ frequenti trasferimenti di olivetani da un monastero all'altro, il loro stile molto simile di decorazione dei manoscritti e il coinvolgimento di diversi pittori-artisti, si evince l'alto livello artistico dei loro manoscritti ${ }^{14}$.

La combinazione di queste circostanze, tuttavia, causa notevoli difficoltà nel determinare il luogo in cui è stato realizzato il libro di Łódź. Gli studi comparativi del codice di Łódź con altri manoscritti olivetani saranno di fondamentale importanza a questo riguardo. Secondo la perizia del Prof.ssa Giordana Mariani Canova dell'Università di Padova, che si occupa dello studio delle miniature nei codici olivetani, è sicuramente un manoscritto italiano della metà del XV secolo (forse anche prima della metà del secolo), molto probabilmente preparato per un monastero olivetano nell'Italia settentrionale. Fu redatto da un monaco che probabilmente fu anche autore delle iniziali decorative realizzate a inchiostro ${ }^{15}$. Queste, invece, rispetto alle miniature dipinte con colori nei codici olivetani, secondo G.M. Canova non sono state ancora adeguatamente analizzate dagli storici dell'arte.

La datazione del manoscritto, in assenza di altri elementi, aiuta anche a determinare lo stile delle iniziali. Il Salterio di Łódź ha iniziali ornamentali di tre tipi, che differiscono per dimensioni e invenzione della realizzazione. Tuttavia, tutte erano scritte solo con inchiostro blu e rosso (Il. 1). Le lettere iniziali più grandi (tipo A) sono le più ricche graficamente e sono state usate per i salmi che iniziano ogni sezione del libro. Il secondo tipo di iniziali (tipo B) è composto dalle lettere molto più piccole, che iniziano la maggior parte dei salmi di una data ora del breviario. L'ultimo tipo di iniziali (tipo C) sono le lettere più semplici e più piccole, usate alternativamente in blu e rosso per indicare i seguenti versi del salmo. Tutte le iniziali hanno delicati elementi decorativi.

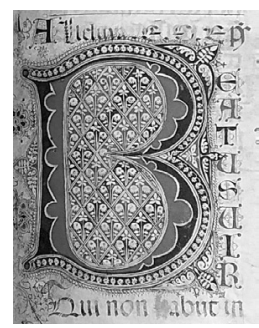

Il. 1.

Tipo A

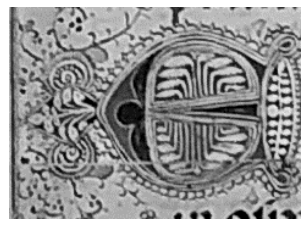

Tipo B

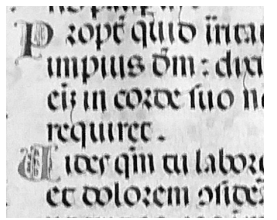

Tipo C

${ }^{13}$ Gli olivetani erano molto spesso scribi e miniaturisti (realizzavano miniature decorative con l'inchiostro), mentre i creatori delle miniature dipinte a colori vivaci erano solitamente miniaturisti laici professionisti, anche se non era sempre così. Vedi G.M. Canova, M. Tagliabue, I corali miniati di Santa Maria di Monte Oliveto: Nuovi documenti e nuove considerazioni, in: Bernardo Tolomei e le origini di Monte Oliveto. Atti del Convegno di studi per il VII centenario di fondazione dell'abbazia (Monte Oliveto Maggiore, 9-10 maggio 2019), ed. G. Andenna, M. Tagliabue, Cesena 2020, p. 323.

${ }^{14}$ Ibidem, p. 324.

${ }^{15}$ G.M. Canova, Corrispondenza e-mail (29.01.2021). 
Alla luce dei manoscritti olivetani da Monte Oliveto conservati nel Museo del Duomo di Chiusi (12 antifonari ${ }^{16}, 6$ graduali ${ }^{17}, 2$ salteri $^{18}$ ), Bologna ${ }^{19}$ e Ferrara ${ }^{20}$, si può affermare che il Salterio di Łódź sicuramente non appartiene a queste raccolte manoscritte ${ }^{21}$. L'alto livello artistico di quei codici, insieme alle composizioni figurali, fioriture marginali o miniature multicolori con l'uso della foglia d'oro, indicano un diverso scriptorium al quale ascrivere l'origine del codice di Łódź.

Il Salterio di Łódź ha una grande somiglianza con il Graduale olivetano (ca. 1430-1439) realizzato per il monastero di San Gerolamo a Quarto vicino Genova o per il Convento di Santa Maria in Baggio vicino a Milano, ora conservato a Yale (USA, Yale University, Beinecke Library, ms. 118422). Un'analisi comparativa del grado di ornamento delle lettere iniziali del Salterio da Lódź con il Graduale ms. 1184 (Il. 2) e un identico ammodernamento delle forme di scrittura di alcuni neumi (quadrati accorciati o allungati ${ }^{23}$ per salvare o riempire lo spazio libero

${ }^{16}$ Museo della Cattedrale, Antifonario A, https://www.museodellacattedrale.it/antifonario-a/; Museo della Cattedrale, Antifonario B, https://www.museodellacattedrale.it/antifonario-b/; Museo della Cattedrale, Antifonario $C$, https://www.museodellacattedrale.it/antifonario-c/; Museo della Cattedrale, Antifonario D, https://www.museodellacattedrale.it/antionario-d/; Museo della Cattedrale, Antifonario E, https://www.museodellacattedrale.it/antifonario-e/; Museo della Cattedrale, Antifonario $F$, https://www.museodellacattedrale.it/antifonario-f/; Museo della Cattedrale, Antifonario H, https://www.museodellacattedrale.it/antifonario-h/; Museo della Cattedrale, Antifonario I, https://www.museodellacattedrale.it/antifonario-i/; Museo della Cattedrale, Antifonario $K$, https://www.museodellacattedrale.it/antifonario-k/; Museo della Cattedrale, Antifonario L, https:// www.museodellacattedrale.it/antifonario-1/; Museo della Cattedrale, Antifonario M, https://www. museodellacattedrale.it/antifonario-m/; Museo della Cattedrale, Antifonario N, https://www.museodellacattedrale.it/antifonario-n/ (accesso: 04.02.2021).

${ }^{17}$ Museo della Cattedrale, Antifonario $O$, https://www.museodellacattedrale.it/antifonario-o/; Museo della Cattedrale, Graduale P, https://www.museodellacattedrale.it/graduale-p/; Museo della Cattedrale, Graduale Q, https://www.museodellacattedrale.it/graduale-q/; Museo della Cattedrale, Graduale Y, https://www.museodellacattedrale.it/graduale-y/; Museo della Cattedrale, Graduale $S$, https://www.museodellacattedrale.it/graduale-s/; Museo della Cattedrale, Graduale T, Museo della Cattedrale, Kyriale-Graduale T, https://www.museodellacattedrale.it/kyriale-e-graduale-t/ (accesso: 04.02.2021).

${ }^{18}$ Museo della Cattedrale, Salterio $U$, https://www.museodellacattedrale.it/salterio-u/; Museo della Cattedrale, Salterio V, https://www.museodellacattedrale.it/salterio-v/ (accesso: 04.02.2021).

${ }^{19}$ Psalterium nocturnale secundum morem monachorum montis Oliveti, http://www.bibliotecamusica.it/cmbm/viewschedatwbca.asp?path=/cmbm/images/ripro/gaspari/_Lit/Lit003/_accesso: 04.02.2021).

${ }^{20}$ I corali oliventani di San Giorgio fuori le mura, https://artsandculture.google.com/ exhibit/i-corali-oliventani-di-san-giorgio-fuori-le-mura/tAIC6HbeU6MeJA (accesso: 04.02.2021).

${ }^{21}$ Canova, Tagliabue, I corali miniati, cit. a nota 13.

${ }^{22}$ Gradual, https://collections.library.yale.edu/catalog/16037197 (accesso: 11.02.2021).

${ }^{23}$ Non hanno significato ritmico. Certamente, alcuni modi di registrare il ritmo in monodia erano conosciuti abbastanza presto (ad esempio l'uso delle configurazioni di notazione come pressus, bistropha, tristropha, allungamento del suono finale, completamento secondario con una linea verticale punctum inclinatum), vedi: T. Maciejewski, Elemety systemu menzuralnego $w$ monodii chorałowej XIII-XVI wieku, in: Notae musicae artis. Notacja muzyczna w źródtach polskich XI-XVI 
sulla quattro righe) restringono l'area geografica dove venne molto probabilmente redatto il codice di Łódź all'Italia settentrionale.

Il. 2.

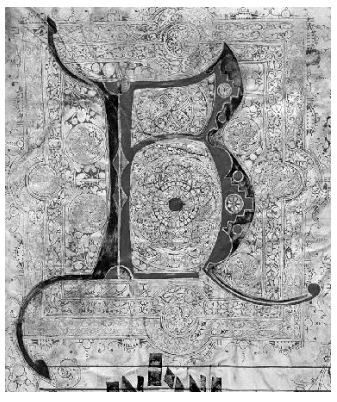

Tipo A

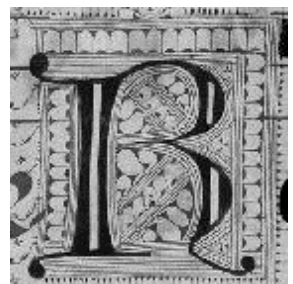

Tipo B

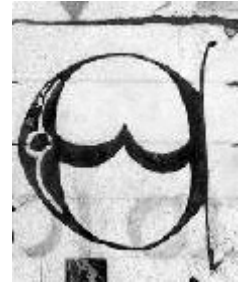

Tipo C

Un importante criterio musicale per identificare i codici liturgici sono le clausole differenziali euouae (saeculorum amen) ${ }^{24}$. Classificare la raccolta di queste formule in un messaggio specifico è un importante strumento scientifico per chiarire la provenienza di una data fonte e per riconoscere la tradizione liturgica e musicale in essa contenuta. Lo scopo della differenziale è combinare esteticamente il tono del salmo con la formula dell'intonazione dell'antifona e decidere inequivocabilmente se la melodia del salmo appartiene a un tono specifico ${ }^{25}$. La pratica di annotare il salmo sotto forma di differenziale era già stata adottata nel trattato Commemoratio brevis della fine del $\mathrm{X}$ secolo $^{26}$. A questo punto, presteremo attenzione solo al modo in cui la formula di parole euouae è scritta nel codice di Łódźz ${ }^{27}$. I codici italiani, a cui appartiene il Salterio di Łódź, conoscono diciotto varianti di scrittura delle sillabe di dossologia saeculorum amen ${ }^{28}$ :

wieku, ed. E. Witkowska-Zaremba, Kraków 1999, p. 296, tuttavia questa pratica non è presente nel Salterio di Łódź.

${ }^{24}$ Nella tradizione gregoriana, la formula differenziale è composta da 6 a 13 note. Molto spesso sotto la notazione di una melodia c'è un'abbreviazione euouae delle ultime parole della piccola dossologia Gloria Patri. Questi due versi dossologici, scritti in Oriente, furono approvati da papa Damaso I (366-384) che ordinò di cantarli alla fine del salmo. Così, ogni salmo terminava con parole identiche, il che rendeva più facile la notazione del testo della formula differenziale con l'abbreviazione euouae, Cz. Grajewski, Dyferencje psalmowe - uwarunkowania historyczne i estetyczne, „Saeculum Christianum”, 12 (2005) n. 1, p. 140.

${ }^{25}$ P. Wagner, Einführung in die gregorianischen Melodien, vol. 3: Gregorianische Formenlehre, Leipzig 1921, p. 129; M. Huglo, Les tonaires. Inventaire, analyse, comparaison, Paris 1971, p. 392. I teorici hanno tentato di creare cataloghi speciali dei finali dei toni del salmo, realizzando elenchi delle antifone con le terminazioni appropriate. La prima attestazione di ciò appare nel trattato Musica disciplina di Aureliano da Réôme (850), The Singing of Psalms in the Early-Medieval Office, „Speculum”, 64 (1989) n. 3, p. 539.

${ }^{26}$ J. Claire, Les répertoires liturgiques latins avant l'octoéchos, „Études Grégoriennes”, 15 (1975) pp. 21-49.

${ }^{27}$ L'analisi delle formule melodiche euouae del codice di Łódź sarà oggetto di uno studio a parte.

${ }^{28}$ B.G. Baroffino, Le differenziae nei codoci Italiani, „Ecclesia Orans” 9 (1992) p. 63. 
I. Seuouae

II. $\mathrm{S}$------ ae

III. Seuo - ae

IV. Se - o-e

V. euouae

VI. euoua

VII. euou - e

VIII. euou

IX. euo - ae

X. euo - e

XI. euo

XII. e - o-ae

XIII. e - o - e

XIV. e - o

XV. e ----- e

XVI. ----- ae

XVII. euorae

XVIII. euououae

Il Salterio di Łódź ha la seguente serie d'iscrizione di dossologia con e la loro distribuzione (52 differenziali in totale):

I. euouae 24

II. e - o-e 18

III. euo - ae 1

IV. e - o- ae 8

V. euou - e 1

La formula euouae (come modello) è una delle più diffuse nei codici italiani. Si ritrova, tra l'altro, nei libri di Ivrea, Montecassino, Napoli ${ }^{29}$. Il differenziale $e$-o-e è presente nei manoscritti di Arezzo, dei monasteri di Montecassino, Napoli, Orvieto, Perugia, Roma e Tropea ${ }^{30}$; euo-ae viene notato nei manoscritti di Arezzo, Orvieto e Pisa ${ }^{31} ;$ e-o-ae è presente nei codici di Montecassino e Perugia ${ }^{32}$, e la clausola euou-e è presente nei codici di Puglia e Bologna ${ }^{33}$. Il Salterio di Łódź conferma la presenza di cinque clausole utilizzate nei codici italiani. Come i libri italiani, il codice usa più volentieri la formula completa euouae (24 volte), in misura leggermente minore $e-o-e$ (18 volte), occasionalmente usa $e$-o-ae (8 volte) e i differenziali III e V solo una volta. Naturalmente, solo l'analisi delle melodie di queste formule porterà a identificare la tradizione liturgica e musicale di questo

\footnotetext{
${ }^{29}$ Ibidem, p. 64.

${ }^{30}$ Ibidem, p. 65.

${ }^{31}$ Ibidem, p. 64.

${ }^{32}$ Ibidem, p. 65.

${ }^{33}$ Ibidem, p. 64.
} 
codice $^{34}$, ma anche la loro forma verbale è un'indicazione che il manoscritto di Łódź utilizza non solo la versione completa (universale) della versione euouae, ma anche varianti incomplete, presenti nei codici italiani.

\section{Fasi del "cammino" del Codice ${ }^{35}$}

Un problema affascinante è determinare la migrazione dei codici manoscritti. Questo fenomeno è noto già nel Medioevo, il che indica il ruolo trascurabile dei confini in quel periodo. Nei manoscritti sono spesso presenti gli elementi chiamati "paratestuali", che consentono di comprendere il testo di un dato codice, pur non essendo parte di esso. Si tratta di miniature, colofoni, poesie di copisti, rubriche, una pagina vivente, "mani", ex libris o commenti ${ }^{36}$. Ė grazie a loro che è molto più facile ricostruire il "viaggio" del manoscritto e determinare i diversi luoghi dove venne utilizzato nella liturgia. Una prova importante dell'adeguamento del libro alle norme liturgiche in vigore, soprattutto alle disposizioni del Tridentinum nel caso dei libri pre-tridentini ${ }^{37}$, sono le note a margine. Tuttavia, nel caso del Salterio di Łódź, libro pre-tridentino, non si ritrovano gli elementi sopra menzionati; a quanto pare, in questo codice, le marginalità non erano necessarie.

Importante per la ricerca è l'incipit del codice di Łódź: „Ad laudem sanctissime trinitatis et beati benedicti abbatis. Incipit psalterium secundum consuetudinem monacorum ordinis monasticis oliveti", che conferma trattarsi di un salterio monastico appartenente agli olivetani della Congregazione Benedettina di Santa Maria del Monte Oliveto (Congregatio Sanctae Mariae Montis Oliveti ${ }^{38}$ ). Questa menzione è una sorta di nota di provenienza del libro, a dimostrazione della recezione del repertorio in un determinato ambiente e area geografica, sebbene i dettagli a essa correlati richiedano un'approfondita ricerca analitica. È noto, quindi, che i primi proprietari del Salterio furono gli olivetani. Alla luce di precedenti rilievi paleografici, si può indicare il nord Italia come luogo di realizzazione del

${ }^{34}$ Le terminazioni dei salmi possono essere un segno distintivo delle tradizioni liturgiche monastiche, ma non sono, tuttavia, di rilievo per stabilire la provenienza in relazione al canto diocesano. Ciò è dovuto agli effetti delle riforme liturgiche attuate da alcuni monasteri nei primi secoli del secondo millennio. I codici europei dal XIII al XIV secolo mostrano generalmente una risorsa differenziale abbastanza uniforme. Si possono distinguere circa 40 tipi melodici ricorrenti di antifone nell'intero del repertorio gregoriano. Vedi Grajewski, Dyferencje psalmowe, pp. 143-145; secondo F. Gevaert, nell'intero repertorio gregoriano si possono distinguere ca. 40 tipi melodici ricorrenti di antifone. Vedi F. Gevaert, Les Origines du chant liturgique de l'Église latine, Ghent 1890, cit. da: Grajewski, Dyferencje psalmowe, p. 145.

${ }^{35}$ Vorrei esprimere il mio ringraziamento al Rev. Kamil Pozorski SDB per il suo aiuto nello stabilire contatti con gli olivetani italiani, la comunità scientifica dell'Università di Padova e preziosi suggerimenti e informazioni su come ricostruire la migrazione del Salterio.

${ }^{36}$ Sosnowski, Tylus, Co mówiq stare rękopisy, pp. 152-155.

${ }^{37}$ Z. Kołodziejczak, Antyfonarz „,De Sanctis” z Biblioteki Kolegiaty w Lasku w świetle tradycji liturgiczno-muzycznej polskiej i europejskiej. Studium źródłoznawczo-muzykologiczne, Warszawa 1994, p. 23 (tesi di dottorato).

${ }^{38}$ G. Piccaso, Congregazione Benedettina Olivetana, w: Dizionario degli Istituti di Perfezione, vol. 2, ed. G. Pelliccia, G. Rocca, Roma 1975, p. 1494; S. Brzozecki, Oliwetanie, in: Encyklopedia katolicka, vol. 14, ed. E. Gigilewicz, Lublin 2010, col. 535-536. 
codice, molto probabilmente destinato a uno dei suddetti monasteri, Quarto ${ }^{39} \mathrm{o} \mathrm{Bag-}$ $\mathrm{gio}^{40}$, sebbene anche questo tema richieda ulteriori approfondimenti. In generale, comunque, lo stile del Salterio dovrebbe essere associato al Graduale del ms. 1184. La decorazione delle lettere iniziali più piccole del codice di Łódź è, a parte lo stile di scrittura, un'importante caratteristica distintiva dello scriptorium o una modalità propria dello scrittore.

Nel nostro caso, si tratta dell'arrivo del manoscritto dall'Italia settentrionale in Polonia. Mentre la provenienza olivetana del Salterio è ovvia, la sua presenza a Łódź lascia perplessi, tanto più che gli olivetani sono quasi sconosciuti nella storiografia polacca. I risultati della ricerca di K. Pozorski mostrano che la congregazione era attestata in Polonia solo da un breve soggiorno di tre monaci (Padre Maurycy Święczkowski ${ }^{41}$, Padre Kazimierz Maria Rygielski ${ }^{42}$, Padre Michelangelo Maria Gala $^{43}$ ) che provenivano dal Monastero di San Giuseppe a Tanzenberg ${ }^{44}$ in Austria (diocesi di Gurk-Klagenfurt) per raggiungere l'abbazia post-cistercense di Ląd sul fiume Warta (1919-1921), dove sarebbero stati loro affidati il monastero e la parrocchia. La decisione della Sacra Congregazione per la Vita Religiosa di erigere il monastero olivetano a Ląd nel 1920, tuttavia, non ha portato al radicarsi in Polonia di una nuova comunità. Già nel gennaio 1921 i monaci chiesero ai propri superiori di lasciare Ląd a causa delle crescenti difficoltà legate ai lavori di ristrutturazione del monastero e ai problemi economici. Con il consenso dell'abate generale degli olivetani Mario Parodia e del vescovo della diocesi di Kuyavian-Kalisz Stanisław Zdzitowiecki, lasciarono Ląd nel marzo $1921^{45}$. È possibile che questi olivetani, venendo in Polonia, portassero con sé il Salterio da Tanzenberg. È noto dalle biografie degli olivetani di Ląd che dopo aver lasciato questo

\footnotetext{
${ }^{39}$ M. Bollati, Un Graduale Olivetano. Un Percorso nella Miniatura Lombarda del Quattrocento, London 2008, pp. 15-17.

${ }^{40}$ La presenza degli olivetani in Lombardia risale all'inizio del XV secolo. Il monastero di Baggio, fondato su iniziativa di Balzarino Pusterla (ca. 1340-1408), fu il primo monastero in Lombardia. Il suo scioglimento avvenne il 12 aprile 1773 e i monaci furono trasferiti al monastero di San Vittore a Milano. Oltre al monastero di Baggio, è documentata l'esistenza di altri monasteri in Lombardia: Santa Maria Incoronata a Nerviano, San Vittore al Corpo a Milano, San Pietro a Civate e San Pietro a Viboldone. Vedi Bollati, Un Graduale Olivetano, pp. 9-10.

${ }^{41}$ Nato il 15 febbraio 1879 a Wenzkau (Germania), completò il noviziato a Seragno vicino a Milano. Emise la sua prima professione religiosa nel 1896, prese i voti perpetui nel 1900 a Tanzenberg e fu ordinato sacerdote nel 1901 a Klagenfurt. Vedi K. Pozorski, Oliwetanie w Ladzie nad Warta 1919-1921, „Polonia Maior Orientalis”, 7 (2020) p. 103.

${ }^{42}$ Nato a Kulm il 23 dicembre 1886 a Kornatowo in Pomerania; fece il noviziato a Seragno vicino a Milano, nel 1905 emise la prima professione religiosa e nel 1908 prese nella basilica i voti perpetui. Ibidem, p. 104.

${ }^{43}$ Nato il 22 febbraio 1891 a Sopornyja (Ungheria); emise la sua prima professione religiosa nel 1911 e prese i voti religiosi nel 1917. Ibidem.

${ }^{44}$ Ch. Cvetko, L'abbazia olivetana di San Giuseppe a Tanzenberg, „L'Ulivo: Rivista olivetana di spiritualita e di cultura monastica”, 1 (2014) p. 72. Il registro dei monaci dell'abbazia di Tanzenberg afferma che almeno alcuni di loro che vivevano nell'Impero austro-ungarico erano di origine polacca: Archivo Abbazia Monte Oliveto Maggiore, Registro dei monaci dell'abbazia di San Giuseppe a Tanzenberg, cit. da: Pozorski, Oliwetanie, p. 102.

${ }^{45}$ Ibidem, pp. 103-106.
} 
monastero ricevettero il permesso di rimanere in Polonia e di entrare nelle fila del clero diocesano ${ }^{46}$.

Relativamente alla presenza del Salterio a Łódź, sembra particolarmente interessante la vicenda di padre Michał Gala, incardinato nella diocesi di Włocławek nel $1924^{47}$. Alla luce della documentazione archivistica, si sa che fu vicario nella parrocchia di Tuszyn ${ }^{48}$, a sua volta passata alla diocesi di Łódź nel 1925. Il parroco di questa parrocchia era il Rev. Stanisław Muznerowski (appassionato storico e collezionista di antichità), la cui biblioteca alla sua morte (1925) arricchì la diocesi di Łódź ${ }^{49}$. È quindi possibile che il Salterio, donato a al Rev. Muznerowski da Padre Gala, sia arrivato a Łódź. In mancanza di informazioni sul Salterio nel Museo di Łódź, questa ipotesi rimane l'unica spiegazione possibile di come il volume sia giunto in Polonia.

L'autore di questo articolo aveva in precedenza considerato altre ipotesi, tra cui quella relativa alla fondazione della regina Bona ${ }^{50}$. Tuttavia, tenendo conto del contesto attualmente delineato, questa e altre ipotesi sembrano piuttosto improbabili.

Indubbiamente il problema rimane ancora aperto e sarà forse possibile ottenere altre informazioni su questo manoscritto nel corso di ulteriori ricerche che si stanno conducendo relative ai temi musicologici che lo riguardano.

Traduzione di Aleksandra Krauze-Kołodziej

\section{REFERENCES / BIBLIOGRAFIA}

\section{Fonti}

I corali oliventani di San Giorgio fuori le mura, https://artsandculture.google.com/exhibit/i-corali-oliventani-di-san-giorgio-fuori-le-mura/tAIC6HbeU6MeJA (accesso: 04.02.2021).

Museo della Cattedrale di Chiusi, Antifonario A, https://www.museodellacattedrale.it/antifonario-a/ (accesso: 04.02.2021).

${ }^{46}$ Il desiderio di rimanere in Polonia è stato dettato, tra gli altri, dalla mancanza di interesse dei superiori per la loro sorte a Ląd. Ciò deriva dalla corrispondenza tra Ląd e Monte Oliveto: Archivio Abbazia Monte Oliveto Maggiore, Korespondencja o. Maura Święczkowskiego do Opata Generalnego o. Mauro Parodi (Cartella Ląd nad Wartą), b.s.; Padre Święczkowski è trasferito all'arcidiocesi di Gniezno, Padre Gala alla diocesi di Włocławek, Padre Rygielski alla diocesi di Katowice: Pozorski, Oliwetanie, p. 105.

${ }^{47}$ Ibidem, p. 105. Il suo cognome apparisce nel registro del 1926 del clero della diocesi di Włocławek. Era un vicario nella parrocchia di Kowal: Catalogus ecclesiarum et utriusque cleri tam saecularis quam regularis Diocesis Wladislaviensis. Pro anno Domini 1926, p. 30.

${ }^{48}$ Catalogus ecclesiarum et utriusque cleri tam saecularis quam regularis Diocesis Wladislaviensis seu Calissiensis. Pro anno Domini 1925, p. 82.

${ }^{49}$ Ibidem; Rev. Antoni Poniński, Corrispendenza e-mail del 3 febbraio 2021.

${ }^{50} \mathrm{Il}$ Museo dell'Arcidiocesi di Łódź conserva un frammento dell'altare di San Nicola di Bari e una casula rossa ricamata decorata con lo stemma della Polonia e degli Sforza: Wiśniewski, Nieznany rękopis liturgiczno-muzyczny. 
Museo della Cattedrale di Chiusi, Antifonario B, https://www.museodellacattedrale.it/antifonario-b/ (accesso: 04.02.2021).

Museo della Cattedrale di Chiusi, Antifonario C, https://www.museodellacattedrale.it/antifonario-c/ (accesso: 04.02.2021).

Museo della Cattedrale di Chiusi, Antifonario D, https://www.museodellacattedrale.it/antionario-d/ (accesso: 04.02.2021).

Museo della Cattedrale di Chiusi, Antifonario E, https://www.museodellacattedrale.it/antifonario-e/ (accesso: 04.02.2021).

Museo della Cattedrale di Chiusi, Antifonario F, https://www.museodellacattedrale.it/antifonario-f/ (accesso: 04.02.2021).

Museo della Cattedrale di Chiusi, Antifonario H, https://www.museodellacattedrale.it/antifonario-h/ (accesso: 04.02.2021).

Museo della Cattedrale di Chiusi, Antifonario I, https://www.museodellacattedrale.it/antifonario-i/ (accesso: 04.02.2021).

Museo della Cattedrale di Chiusi, Antifonario K, https://www.museodellacattedrale.it/antifonario-k/ (accesso: 04.02.2021).

Museo della Cattedrale di Chiusi, Antifonario L, https://www.museodellacattedrale.it/antifonario-1/ (accesso: 04.02.2021).

Museo della Cattedrale di Chiusi, Antifonario M, https://www.museodellacattedrale.it/antifonario-m/ (accesso: 04.02.2021).

Museo della Cattedrale di Chiusi, Antifonario N, https://www.museodellacattedrale.it/antifonario-n/ (accesso: 04.02.2021).

Museo della Cattedrale di Chiusi, Graduale O, https://www.museodellacattedrale.it/antifonario-o/ (accesso: 04.02.2021).

Museo della Cattedrale di Chiusi, Graduale P, https://www.museodellacattedrale.it/graduale-p/ (accesso: 04.02.2021).

Museo della Cattedrale di Chiusi, Graduale Q, https://www.museodellacattedrale.it/graduale-q/ (accesso: 04.02.2021).

Museo della Cattedrale di Chiusi, Graduale Y, https://www.museodellacattedrale.it/graduale-y/ (accesso: 04.02.2021).

Museo della Cattedrale di Chiusi, Graduale S, https://www.museodellacattedrale.it/graduale-s/ (accesso: 04.02.2021).

Museo della Cattedrale di Chiusi, Kyriale-Graduale T, https://www.museodellacattedrale. it/kyriale-e-graduale-t/ (accesso: 04.02.2021).

Museo della Cattedrale di Chiusi, Salterio U, https://www.museodellacattedrale.it/salterio-u/ (accesso: 04.02.2021).

Museo della Cattedrale di Chiusi, Salterio V, https://www.museodellacattedrale.it/salterio-v/ (accesso: 04.02.2021).

Museo Internazionale e Biblioteca della Musica, Bologna, Psalterium nocturnale secundum morem monachorum montis Oliveti, http://www.bibliotecamusica.it/cmbm/viewschedatwbca.asp?path=/cmbm/images/ripro/gaspari/_Lit/Lit003/(accesso: 04.02.2021).

Yale University, Beinecke Library, ms. 1184, https://collections.library.yale.edu/cata$\log / 16037197$ (accesso: 11.02.2021).

Archivio Abbazia Monte Oliveto Maggiore, Korespondencja o. Maura Święczkowskiego do Opata Generalnego o. Mauro Parodi (Teczka Ląd nad Wartą), b.s.

Catalogus ecclesiarum et utriusque cleri tam saecularis quam regularis Diocesis Wladislaviensis. Pro anno Domini 1926.

Catalogus ecclesiarum et utriusque cleri tam saecularis quam regularis Diocesis Wladislaviensis seu Calissiensis. Pro anno Domini 1925. 


\section{Pubblicazioni}

Baroffino Bonifacio Giacomo, Le differenziae nei codoci Italiani, „Ecclesia Orans”, 9 (1992) pp. 61-68.

Birkenmajer Aleksander (ed.), Encyklopedia wiedzy o książe, Wrocław 1971.

Bisztyga Jerzy, Psalterium Andrzeja Piotrkowczyka z 1599 roku jako pierwszy dokument potrydenckiego choratu gregoriańskiego w Polsce, Lublin 2009 (tesi di dottorato).

Bollati Milvia, Un Graduale Olivetano. Un Percorso nella Miniatura Lombarda del Quattrocento, London 2008.

Bonniwell William, Historia liturgii dominikańskiej 1215-1945 (tłum. D. Krupińska), New York 1945, www.rytdominikanski.pl/historia-liturgii-dominikanskiej/historia-liturgii-dominikanskiej-1215-1945-o-william-bonniwell-op (accesso: 20.11.2020).

Brzozecki Sławomir, Oliwetanie, in: Encyklopedia katolicka, vol. 14, ed. E. Gigilewicz, Lublin 2010, col. 535-536.

Canova Giordana Mariani, Tagliabue Mauro, I corali miniati di Santa Maria di Monte Oliveto: Nuovi documenti e nuove considerazioni, in: Bernardo Tolomei e le origini di Monte Oliveto. Atti del Convegno di studi per il VII centenario di fondazione dell'abbazia (Monte Oliveto Maggiore, 9-10 maggio 2019), ed. G. Andenna, M. Tagliabue, Cesena 2020, pp. 323-396.

Claire Jean, Les répertoires liturgiques latins avant l'octoéchos, „Études Grégoriennes”, 15 (1975) pp. 21-49.

Cvetko Christian, L'abbazia olivetana do San Giuseppe a Tanzenberg, „L'Ulivo: Rivista olivetana di spiritualita e di cultura monastica", 1 (2014) pp. 72-109.

Deyer Joseph, The Singing of Psalms in the Early-Medieval Office, „Speculum”, 64 (1989) n. 3, pp. 535-578.

Gevaert François, Les Origines du chant liturgique de l'Église latine, Ghent 1890.

Grajewski Czesław, Dyferencje psalmowe - uwarunkowania historyczne i estetyczne, „Saeculum Christianum", 12 (2005) n. 1, pp. 139-146.

Hughes Andrew, Medieval Manuscripts for Mass and Office. A guide to their organization and terminology, Toronto 1982.

Huglo Michel, Les tonaires. Inventaire, analyse, comparaison, Paris 1971.

Kołodziejczak Zenon, Antyfonarz „De Sanctis” z Biblioteki Kolegiaty w Łasku w świetle tradycji liturgiczno-muzycznej polskiej i europejskiej. Studium źródłoznawczo-muzykologiczne, Warszawa 1994 (tesi di dottorato).

Kopeć Jerzy, Collatio, in: Encyklopedia katolicka, vol. 3, ed. R. Łukaszyk, L. Bieńkowski, F. Gryglewicz, Lublin 1979, col. 540.

Maciejewski Tadeusz, Elemety systemu menzuralnego $w$ monodii chorałowej XIII-XVI wieku, in: Notae musicae artis. Notacja muzyczna w źródłach polskich XI-XVI wieku, ed. E. Witkowska-Zaremba, Kraków 1999, pp. 283-347.

Pawlak Ireneusz, Hymnarz, in: Encyklopedia katolicka, vol. 6, ed. J. Walkusz, Lublin 1993, col. 1364-1365.

Piccaso Giorgio, Congregazione Benedettina Olivetana, in: Dizionario degli Istituti di Perfezione, vol. 2, ed. G. Pelliccia, G. Rocca, Roma 1975, p. 1494.

Pozorski Kamil, Oliwetanie w Lądzie nad Warta 1919-1921, „Polonia Maior Orientalis”, 7 (2020) pp. 99-108.

Semkowicz Władysław, Paleografia łacińska, Kraków 2002.

Sosnowski Roman, Tylus Piotr, Co mówia stare rękopisy, Kraków 2010.

Wagner Peter, Einführung in die gregorianischen Melodien, vol. 3: Gregorianische Formenlehre, Leipzig 1921. 
Wiśniewski Piotr, Nieznany rękopis liturgiczno-muzyczny z Muzeum Archidiecezji Łódzkiej, „Muzyka”, (2021), in stampa.

\title{
Relazioni
}

Giordana Mariani Canova, Corrispondenza e-mail del 29 gennaio 2021.

Rev. Antoni Poniński, Corrispondenza e-mail del 3 marzo 2021.

\section{RĘKOPIŚMIENNY PSALTERZ PROWENIENCJI OLIWETAŃSKIEJ W POLSCE}

\section{Streszczenie}

Artykuł prezentuje wyniki badań nad księgą rękopiśmiennego Psałterza proweniencji oliwetańskiej przechowywanego w Muzeum Archidiecezji Łódzkiej. Autor podaje syntetyczny opis kodeksu, weryfikuje czas jego powstania, precyzuje obszar geograficzny, w którym został on napisany, oraz wskazuje hipotetyczne miejsce jego użytkowania. W świetle analizy porównawczej Psatterza z innymi rękopisami oliwetańskimi dowodzi, że napisał go oliwetanin będący również autorem dekoracji kodeksu. Końcowa część artykułu jest próbą wyjaśnienia migracji Psałterza z Włoch do Polski.

Słowa kluczowe: psałterz; oliwetanie; Monte Oliveto; Łódź; officium divinum

\section{THE HANDWRITTEN PSALTER OF THE OLIVETAN PROVENANCE IN POLAND}

\begin{abstract}
The article presents the results of research on the book of the handwritten Psalter of the olivetan provenance, stored in the Museum of the Archdiocese of Łódź. The author provides a synthetic description of the code, verifies the time of its origin, specifies the geographical area in which it was written and indicates the hypothetical place of its use. In the light of a comparative analysis of the Psalter with other olivetan manuscripts, it proves that it was written by an olivetan who also made the decorations of the codex. The final part of the article is an attempt to explain the migration of the Psalter from Italy to Poland.
\end{abstract}

Keywords: Psalter; olivetans; Monte Oliveto; Łódź; officium divinum

Translated by Mark Ó. Fionnáin 